\title{
POSTPROCESSING FOR GUARANTEED ERROR BOUND BASED ON EQUILIBRATED FLUXES
}

\author{
KWANG-YEON KIM
}

\begin{abstract}
In this work we analyze a postprocessing scheme for improving the guaranteed error bound based on the equilibrated fluxes for the $P 1$ conforming FEM. The improved error bound is shown to be asymptotically exact under suitable conditions on the triangulations and the regularity of the true solution. We also present some numerical results to illustrate the effect of the postprocessing scheme.
\end{abstract}

\section{Introduction}

In this work we consider the second-order elliptic equation

$$
-\Delta u+c u=f \quad \text { in } \Omega
$$

subject to the boundary condition

$$
u=u_{D} \quad \text { on } \Gamma_{D} \quad \text { and } \quad \frac{\partial u}{\partial n}=g_{N} \quad \text { on } \Gamma_{N},
$$

where $\Omega$ is a bounded polygonal domain in $\mathbb{R}^{2}$ with $\partial \Omega=\overline{\Gamma_{D} \cup \Gamma_{N}}, \Gamma_{D} \cap \Gamma_{N}=$ $\emptyset$, and $c \geq 0$ is a bounded function on $\Omega$. When $\Gamma_{D}=\emptyset$, we assume that $c>0$ in a subdomain of $\Omega$ having a positive measure. Moreover, $c$ is supposed to be of moderate size, i.e., we do not consider the singularly perturbed case.

Given some finite element approximation $u_{h} \approx u$, the unknown error $e:=$ $u-u_{h}$ is estimated by an error estimator which is computable a posteriori in terms of the numerical solution and the given problem data. There are currently a number of error estimators available in the literature (see the monographs $[2,3]$ for a complete discussion), most of which are reliable and efficient in the sense that the following upper and lower bounds hold for the estimated error

Received October 17, 2014; Revised March 10, 2015.

2010 Mathematics Subject Classification. Primary 65N30, 65N15.

Key words and phrases. a posteriori error estimation, equilibrated fluxes, guaranteed error bound, asymptotic exactness, superconvergence.

This research was supported by Basic Science Research Program through the National Research Foundation of Korea (NRF) funded by the Ministry of Education, Science and Technology (2011-0023269). 
$\eta$ and the actual error in the energy norm $\|\cdot\|$

$$
C_{1} \eta+\text { (h.o.t) } \leq\|e\| \leq C_{2} \eta+\text { (h.o.t), }
$$

where the positive constants $C_{1}, C_{2}$ are independent of the mesh size and (h.o.t) represents a higher order term. Some error estimators produce surprisingly accurate results by exhibiting the so-called asymptotic exactness

$$
\|e\|=\eta+\text { (h.o.t) } \quad \text { or } \quad \lim _{h \rightarrow 0} \frac{\eta}{\|e\|}=1
$$

under favorable conditions which ensure some superconvergence result (see, for example, $[4,5,11,12,13,15,17,18,22,23])$.

In recent years, there has been an intensive study of the error estimators providing the guaranteed error bound

$$
\|e\| \leq \eta\left(\boldsymbol{\sigma}_{h}\right)+\text { (h.o.t) }
$$

based on recovery of the $H(\operatorname{div})$-conforming vector approximation $\boldsymbol{\sigma}_{h} \approx \boldsymbol{\sigma}=$ $\nabla u$ (a specific form of $\eta\left(\boldsymbol{\sigma}_{h}\right)$ is given in (8) below). We refer to $[1,7,10,16$, $19,20,21]$ for recent results on conforming FEMs and [14] for earlier results. Numerical experiments show that in many cases the effectivity index $\eta\left(\boldsymbol{\sigma}_{h}\right) /\|e\|$ lies below 2, but it was explained in [8] that there exists a threshold between $\eta\left(\boldsymbol{\sigma}_{h}\right) /\|e\|$ and 1 which limits the accuracy of $\eta\left(\boldsymbol{\sigma}_{h}\right)$. One way of improving the accuracy of $\eta\left(\boldsymbol{\sigma}_{h}\right)$ is to add a correction term $\operatorname{curl} \varphi_{h}$ and make $\eta\left(\boldsymbol{\sigma}_{h}+\operatorname{curl} \varphi_{h}\right)$ as small as possible over a set of properly chosen trial functions $\varphi_{h} \in H^{1}(\Omega)$ such that $\left.\varphi_{h}\right|_{\Gamma_{N}}=0$ (cf. $[8,10,20]$ ). This postprocessing was explored in much detail in [8], where $\varphi_{h}$ is chosen to be finite element functions of the same degree as $u_{h}$ over successively red-refined triangulations, and proved to be very effective for several choices of $\boldsymbol{\sigma}_{h}$ available in literature.

In this paper we consider the same postprocessing over a different set of finite element functions $\varphi_{h}$ for the $P 1$ conforming FEM. The vector approximation $\boldsymbol{\sigma}_{h}$ is constructed in the lowest-order Raviart-Thomas space using the piecewise linear equilibrated fluxes of Ainsworth and Oden [2]. Then we seek the optimal function $\psi_{h}$ which minimizes $\eta\left(\boldsymbol{\sigma}_{h}+\operatorname{curl} \varphi_{h}\right)$ over the set of continuous piecewise quadratic bump functions $\varphi_{h}$ (defined on the same triangulation as $u_{h}$ ) such that $\left.\varphi_{h}\right|_{\Gamma_{N}}=0$. This gives rise to a global but well-conditioned SPD matrix system which can be efficiently solved by the conjugate gradient method (with no or the simple diagonal preconditioner). Actually, only a few cg iterations are sufficient to gain significant improvement as observed by numerical results of [8] and Section 5 of this paper.

The idea of choosing higher order functions for $\psi_{h}$ was also mentioned in [8], but not further discussed. In this paper we will prove that the guaranteed error bound $\eta\left(\boldsymbol{\sigma}_{h}+\mathbf{c u r l} \psi_{h}\right)$ is asymptotically exact under the same conditions on the triangulations and the regularity of the solution $u$ as for the gradient recovery error estimator [22] and the Bank-Weiser error estimator [17]. As is usual, the proof depends on the superconvergence property of $\boldsymbol{\sigma}_{h}$. Using the results of [17], we will first show that $\boldsymbol{\sigma}_{h}$ is super-close to the Fortin projection 
of $\sigma$, and then deduce from this that the postprocessed vector approximation $\boldsymbol{\sigma}_{h}+\operatorname{curl} \psi_{h}$ converges to $\boldsymbol{\sigma}$ in the $L^{2}$ norm at a higher rate than $\nabla u_{h}$.

The remainder of the paper is organized as follows. In the next section we introduce some notation and preliminary results on the error estimator yielding the guaranteed error bound. In Section 3 we establish the supercloseness between $\boldsymbol{\sigma}_{h}$ and the Fortin projection of $\boldsymbol{\sigma}$. In Section 4 we present the postprocessing scheme for computing the optimal function $\psi_{h}$ and then derive the asymptotic exactness of the improved error bound $\eta\left(\boldsymbol{\sigma}_{h}+\operatorname{curl} \psi_{h}\right)$. Finally, some numerical results are reported in Section 5 to confirm the theoretical results and illustrate the performance of $\eta\left(\boldsymbol{\sigma}_{h}+\operatorname{curl} \psi_{h}\right)$.

\section{Preliminaries}

We use the standard notation $W^{m, p}(G)$ and $\|\cdot\|_{m, p, G}$ for the Sobolev spaces and their norms over a domain $G \subset \mathbb{R}^{n}(n=1,2)$, with $H^{m}(G)=W^{m, p}(G)$ and $\|\cdot\|_{m, p, G}=\|\cdot\|_{m, G}$ when $p=2$. The $L^{2}$ inner product and norm over $G$ are denoted by $(\cdot, \cdot)_{G}$ and $\|\cdot\|_{0, G}$, respectively.

Let $\left\{\mathcal{T}_{h}\right\}_{h>0}$ be a shape-regular family of triangulations of $\Omega$ in the usual sense. For each triangulation $\mathcal{T}_{h}$, the mesh size is defined as $h=\max _{K \in \mathcal{T}_{h}} h_{K}$. The set of all edges of $\mathcal{T}_{h}$ is denoted by $\mathcal{E}_{h}$, and we set

$$
\mathcal{E}_{D}=\left\{\gamma \in \mathcal{E}_{h}: \gamma \subset \Gamma_{D}\right\}, \mathcal{E}_{N}=\left\{\gamma \in \mathcal{E}_{h}: \gamma \subset \Gamma_{N}\right\}, \mathcal{E}_{I}=\mathcal{E}_{h} \backslash\left(\mathcal{E}_{D} \cup \mathcal{E}_{N}\right) .
$$

For an element $K \in \mathcal{T}_{h}$, the diameter of $K$ is denoted by $h_{K}$ and the set of three edges of $K$ by $\mathcal{E}_{K}$. Throughout the paper, $C$ will denote a generic positive constant independent of the mesh size $h$ which may assume different values at different places. We also use the notation $P_{r}(K)$ to denote the space of all polynomials on $K$ of total degree less than or equal to $r \geq 0$.

The variational formulation of the model problem $(1)-(2)$ is stated as follows: find $u \in H^{1}(\Omega)$ such that $\left.u\right|_{\Gamma_{D}}=u_{D}$ and

$$
B(u, v)=(f, v)_{\Omega}+\int_{\Gamma_{N}} g_{N} v d s \quad \forall v \in H_{D}^{1}(\Omega),
$$

where

$$
B(u, v)=\sum_{K \in \mathcal{T}_{h}} B_{K}(u, v), \quad B_{K}(u, v)=(\nabla u, \nabla v)_{K}+(c u, v)_{K}
$$

and

$$
H_{D}^{1}(\Omega)=\left\{v \in H^{1}(\Omega):\left.v\right|_{\Gamma_{D}}=0\right\} .
$$

The energy norm over a subdomain $G \subset \Omega$ is defined as

$$
\|v\|_{G}:=\left(\sum_{K \subset G} B_{K}(v, v)\right)^{1 / 2}=\left(\|\nabla v\|_{0, G}^{2}+\left\|c^{1 / 2} v\right\|_{0, G}^{2}\right)^{1 / 2} .
$$

For ease of presentation, we will henceforth assume that the Dirichlet datum $u_{D}$ is continuous and piecewise linear over $\mathcal{E}_{D}$ and the Neumann datum $g_{N}$ is piecewise constant over $\mathcal{E}_{N}$. Subsequent results are easily extended to more 
general data, involving only additional higher order perturbations if $u_{D}$ and $g_{N}$ are piecewise smooth.

Let $u_{h}$ be the continuous piecewise linear finite element approximation of the problem (4) over the triangulation $\mathcal{T}_{h}$ such that $\left.u_{h}\right|_{\Gamma_{D}}=u_{D}$. Following Chapter 6 of [2], we construct approximate boundary fluxes $\left\{g_{K} \approx \frac{\partial u}{\partial n_{K}}\right\}_{K \in \mathcal{T}_{h}}$ satisfying the first-order equilibration conditions

$$
\left\{\begin{aligned}
\left(f, v_{h}\right)_{K}-B_{K}\left(u_{h}, v_{h}\right)+\int_{\partial K} g_{K} v_{h} d s & =0 & & \forall v_{h} \in P_{1}(K), \forall K \in \mathcal{T}_{h} \\
g_{K}+g_{K^{\prime}} & =0 & & \text { on } \partial K \cap \partial K^{\prime} \\
g_{K} & =g_{N} & & \text { on } \partial K \cap \Gamma_{N} .
\end{aligned}\right.
$$

The approximate boundary flux $g_{K}$ is taken to be a linear function on every edge of $\mathcal{E}_{D} \cup \mathcal{E}_{I}$. By a clever choice of degrees of freedom the construction can be done by solving small local problems on patches of elements around the vertices of $\mathcal{T}_{h}$; see [2] for more details.

Once the equilibrated fluxes $\left\{g_{K} \in L^{2}(\partial K)\right\}_{K \in \mathcal{T}_{h}}$ are found, we can locally compute a vector approximation $\boldsymbol{\sigma}_{h} \approx \boldsymbol{\sigma}=\nabla u$ in the lowest-order RaviartThomas space

$$
\mathbb{R} \mathbb{T}_{0}(K):=\left(P_{0}(K)\right)^{2} \oplus(x, y) P_{0}(K)
$$

by specifying the normal component of $\left.\boldsymbol{\sigma}_{h}\right|_{K}$ on $\partial K$ with

$$
\left.\int_{\gamma} \boldsymbol{\sigma}_{h}\right|_{K} \cdot \boldsymbol{n}_{K} d s=\int_{\gamma} g_{K} d s \quad \forall \gamma \in \mathcal{E}_{K},
$$

where $\boldsymbol{n}_{K}$ denotes a unit normal outward to $K$. By virtue of the equilibration conditions (5), $\boldsymbol{\sigma}_{h}$ has continuous normal components across edges of $\mathcal{T}_{h}$ and satisfies the local conservation law

$$
\left(\operatorname{div} \boldsymbol{\sigma}_{h}+f-c u_{h}, 1\right)_{K}=0 \quad \forall K \in \mathcal{T}_{h} .
$$

We can then obtain the following guaranteed error bound by straightforward extension of $[1,7,10,16,21]$ to the case of nonzero $c$ of moderate size

(8) $\left\|u-u_{h}\right\|_{\Omega} \leq\left\{\sum_{K \in \mathcal{T}_{h}}\left(\left\|\boldsymbol{\sigma}_{h}-\nabla u_{h}\right\|_{0, K}+\frac{h_{K}}{\pi}\left\|\operatorname{div} \boldsymbol{\sigma}_{h}+f-c u_{h}\right\|_{0, K}\right)^{2}\right\}^{1 / 2}$.

Numerical results show that the error bound (8) is not asymptotically exact even when the triangulation $\mathcal{T}_{h}$ is uniform and the solution $u$ is smooth.

Before closing this section, we define the global Raviart-Thomas and BrezziDouglas-Marini spaces over $\mathcal{T}_{h}$ by

$$
\begin{aligned}
\mathbb{R T}_{0} & =\left\{\boldsymbol{\tau} \in H(\operatorname{div} ; \Omega):\left.\boldsymbol{\tau}\right|_{K} \in \mathbb{R T}_{0}(K) \quad \forall K \in \mathcal{T}_{h}\right\}, \\
\mathbb{B D M}_{1} & =\left\{\boldsymbol{\tau} \in H(\operatorname{div} ; \Omega):\left.\boldsymbol{\tau}\right|_{K} \in\left(P_{1}(K)\right)^{2} \quad \forall K \in \mathcal{T}_{h}\right\},
\end{aligned}
$$

where

$$
H(\operatorname{div} ; \Omega)=\left\{\boldsymbol{\tau} \in\left(L^{2}(\Omega)\right)^{2}: \operatorname{div} \boldsymbol{\tau} \in L^{2}(\Omega)\right\}
$$


Note that $\boldsymbol{\sigma}_{h} \in \mathbb{R T}_{0} \subset \mathbb{B D M}_{1}$ although it is computed locally on each element of $\mathcal{T}_{h}$. We also define the Fortin projections $\Pi_{h}:\left(H^{1}(\Omega)\right)^{2} \rightarrow \mathbb{R} \mathbb{T}_{0}$ by

$$
\int_{\gamma} \Pi_{h} \boldsymbol{\tau} \cdot \boldsymbol{n}_{\gamma} d s=\int_{\gamma} \boldsymbol{\tau} \cdot \boldsymbol{n}_{\gamma} d s \quad \forall \gamma \in \mathcal{E}_{h}
$$

and $\Pi_{h}^{1}:\left(H^{1}(\Omega)\right)^{2} \rightarrow \mathbb{B D M}_{1}$ by

$$
\int_{\gamma} \Pi_{h}^{1} \boldsymbol{\tau} \cdot \boldsymbol{n}_{\gamma} \mu d s=\int_{\gamma} \boldsymbol{\tau} \cdot \boldsymbol{n}_{\gamma} \mu d s \quad \forall \mu \in P_{1}(\gamma), \forall \gamma \in \mathcal{E}_{h},
$$

where $\boldsymbol{n}_{\gamma}$ denotes a unit normal to $\gamma$. It is well known that

$$
\left\|\boldsymbol{\sigma}-\Pi_{h} \boldsymbol{\sigma}\right\|_{0, \Omega} \leq C h\|\boldsymbol{\sigma}\|_{1, \Omega}, \quad\left\|\boldsymbol{\sigma}-\Pi_{h}^{1} \boldsymbol{\sigma}\right\|_{0, \Omega} \leq C h^{2}\|\boldsymbol{\sigma}\|_{2, \Omega} .
$$

\section{Superconvergence result}

In this section we will establish the super-closeness between the vector approximation $\boldsymbol{\sigma}_{h}$ and the Fortin projection $\Pi_{h} \boldsymbol{\sigma}$ of $\boldsymbol{\sigma}=\nabla u$ assuming that $u \in H^{3}(\Omega) \cap W^{2, \infty}(\Omega)$. Using the scaling argument and the definitions (6) and (9), we find that for every $K \in \mathcal{T}_{h}$,

$$
\begin{aligned}
\left\|\boldsymbol{\sigma}_{h}-\Pi_{h} \boldsymbol{\sigma}\right\|_{0, K}^{2} & \leq C h_{K}\left\|\left(\boldsymbol{\sigma}_{h}-\Pi_{h} \boldsymbol{\sigma}\right) \cdot \boldsymbol{n}_{K}\right\|_{0, \partial K}^{2} \\
& \leq C \sum_{\gamma \in \mathcal{E}_{K}}\left|\int_{\gamma} \boldsymbol{\sigma}_{h} \cdot \boldsymbol{n}_{K} d s-\int_{\gamma} \boldsymbol{\sigma} \cdot \boldsymbol{n}_{K} d s\right|^{2} \\
& =C \sum_{\gamma \in \mathcal{E}_{K}}\left|\int_{\gamma}\left(g_{K}-\frac{\partial u}{\partial n_{K}}\right) d s\right|^{2} .
\end{aligned}
$$

Thus we need to estimate the flux error $\int_{\gamma}\left(g_{K}-\frac{\partial u}{\partial n_{K}}\right) d s$ for $\gamma \in \mathcal{E}_{K}$.

Lemma 3.1. Assume that $u \in H^{2}(\Omega)$ and let $u_{I}$ be the standard nodal interpolant of $u$. Then there exists a constant $C>0$ such that for every $K \in \mathcal{T}_{h}$,

$$
\sum_{\gamma \in \mathcal{E}_{K}}\left|\int_{\gamma}\left(g_{K}-\frac{\partial u}{\partial n_{K}}\right) d s\right| \leq C\left\|u_{h}-u_{I}\right\|_{\omega_{K}}+C h_{K}|u|_{2, \omega_{K}}
$$

where $\omega_{K}=\bigcup\left\{K^{\prime} \in \mathcal{T}_{h}: \bar{K} \cap \overline{K^{\prime}} \neq \emptyset\right\}$.

Proof. Using the Cauchy-Schwarz inequality and then the triangle inequality, we obtain

$$
\begin{aligned}
& \sum_{\gamma \in \mathcal{E}_{K}}\left|\int_{\gamma}\left(g_{K}-\frac{\partial u}{\partial n_{K}}\right) d s\right| \\
\leq & C h_{K}^{1 / 2}\left(\left\|g_{K}-\left\langle\frac{\partial u_{h}}{\partial n_{K}}\right\rangle\right\|_{0, \partial K}+\left\|\left\langle\frac{\partial u_{h}}{\partial n_{K}}\right\rangle-\frac{\partial u}{\partial n_{K}}\right\|_{0, \partial K}\right),
\end{aligned}
$$


where

$$
\left\langle\frac{\partial u_{h}}{\partial n_{K}}\right\rangle= \begin{cases}\frac{1}{2}\left(\left.\nabla u_{h}\right|_{K}+\left.\nabla u_{h}\right|_{K^{\prime}}\right) \cdot \boldsymbol{n}_{K} & \text { on } \partial K \cap \partial K^{\prime} \\ \left.\nabla u_{h}\right|_{K} \cdot \boldsymbol{n}_{K} & \text { on } \partial K \cap \Gamma_{D} \\ g_{N} & \text { on } \partial K \cap \Gamma_{N}\end{cases}
$$

The first term is handled by Theorem 6.2 of [2] and the equality $f=-\Delta u+c u$ :

$$
\begin{aligned}
h_{K}^{1 / 2}\left\|g_{K}-\left\langle\frac{\partial u_{h}}{\partial n_{K}}\right\rangle\right\|_{0, \partial K} & \leq C\left\|u-u_{h}\right\|_{\omega_{K}}+C h_{K}\left\|f-c u_{h}\right\|_{0, \omega_{K}} \\
& \leq C\left\|u-u_{h}\right\|_{\omega_{K}}+C h_{K}|u|_{2, \omega_{K}} \\
& \leq C\left\|u_{I}-u_{h}\right\|_{\omega_{K}}+C h_{K}|u|_{2, \omega_{K}} .
\end{aligned}
$$

For the second term we use the equality $\left.\left\langle\frac{\partial u}{\partial n_{K}}\right\rangle\right|_{\partial K}=\left.\frac{\partial u}{\partial n_{K}}\right|_{\partial K}$ and the trace inequality to obtain

$$
\begin{aligned}
h_{K}^{1 / 2}\left\|\left\langle\frac{\partial u_{h}}{\partial n_{K}}\right\rangle-\frac{\partial u}{\partial n_{K}}\right\|_{0, \partial K} & \leq C\left|u-u_{h}\right|_{1, \omega_{K}}+C h_{K}|u|_{2, \omega_{K}} \\
& \leq C\left\|u_{I}-u_{h}\right\|_{\omega_{K}}+C h_{K}|u|_{2, \omega_{K}} .
\end{aligned}
$$

This completes the proof.

Lemma 3.1 only gives $\left\|\boldsymbol{\sigma}_{h}-\Pi_{h} \boldsymbol{\sigma}\right\|_{0, \Omega}=O(h)$. We need a stronger version of Lemma 3.1 in order to derive the super-closeness. For this purpose we assume that the triangulation $\mathcal{T}_{h}$ satisfies Condition $(\alpha, \sigma)$ introduced in [22]: there exists a partition $\mathcal{T}_{h}=\mathcal{T}_{1, h} \cup \mathcal{T}_{2, h}$ and positive constants $\alpha, \sigma$ such that

(A1) every two adjacent elements of $\mathcal{T}_{1, h}$ form an $O\left(h^{1+\alpha}\right)$ parallelogram (i.e., the lengths of any two opposite edges differ only by $O\left(h^{1+\alpha}\right)$ );

(A2) $\sum_{K \in \mathcal{T}_{2, h}}|K|=O\left(h^{\sigma}\right)$, where $|K|$ denotes the area of $K$.

Let $\Omega_{1, h}=\bigcup_{K \in \mathcal{T}_{1, h}} K$ and define a subset of $\mathcal{T}_{1, h}$ by

$$
\mathcal{T}_{0, h}=\left\{K \in \mathcal{T}_{1, h}: \omega_{K} \subset \Omega_{1, h} \text { and } B(K) \subset \Omega\right\},
$$

where $B(K)$ is a ball of radius $3 h$ containing $\omega_{K}$. Note that we have

$$
\sum_{K \in \mathcal{T}_{h} \backslash \mathcal{T}_{0, h}}|K|=O\left(h^{\min (\sigma, 1)}\right)
$$

by the assumption (A2).

Lemma 3.2. Assume that $u \in H^{3}(\Omega)$. Then there exists a constant $C>0$ such that for every $K \in \mathcal{T}_{0, h}$,

$$
\sum_{\gamma \in \mathcal{E}_{K}}\left|\int_{\gamma}\left(g_{K}-\frac{\partial u}{\partial n_{K}}\right) d s\right| \leq C\left\|u_{h}-u_{I}\right\|_{\omega_{K}}+C h_{K}^{1+\min (\alpha, 1)}\|u\|_{3, B(K)} .
$$


Proof. Let $P_{2}^{0}(K)$ be the space of quadratic polynomials on $K$ that vanish at the vertices of $K$ and let $\phi_{K} \in P_{2}^{0}(K)$ be the solution of the local problem

$$
B_{K}\left(\phi_{K}, v\right)=(f, v)_{K}-B_{K}\left(u_{h}, v\right)+\int_{\partial K} g_{K} v d s \quad \forall v \in P_{2}^{0}(K) .
$$

The following estimate is given in (6.22) of [17]

$$
\left\|e-\phi_{K}\right\|_{K} \leq C\left\|u_{h}-u_{I}\right\|_{\omega_{K}}+C h_{K}^{1+\min (\alpha, 1)}\|u\|_{3, B(K)}
$$

for every $K \in \mathcal{T}_{0, h}$. This will be used at the last step of the proof.

Substituting $f=-\Delta u+c u$ in (11) and then integrating by parts, we obtain for $v \in P_{2}^{0}(K)$

$$
B_{K}\left(\phi_{K}, v\right)=B_{K}\left(u-u_{h}, v\right)+\int_{\partial K}\left(g_{K}-\frac{\partial u}{\partial n_{K}}\right) v d s
$$

and hence

(13) $\int_{\partial K}\left(g_{K}-Q_{1} \frac{\partial u}{\partial n_{K}}\right) v d s=B_{K}\left(\phi_{K}-e, v\right)+\int_{\partial K}\left(\frac{\partial u}{\partial n_{K}}-Q_{1} \frac{\partial u}{\partial n_{K}}\right) v d s$, where $\left.Q_{1} w\right|_{\gamma}$ is the $L^{2}$ projection of $\left.w\right|_{\gamma}$ into $P_{1}(\gamma)$ for $\gamma \in \mathcal{E}_{K}$.

Let $\theta_{\gamma} \in P_{2}^{0}(K)$ be such that $\theta_{\gamma}\left(m_{\gamma}\right)=1$ and $\left.\theta_{\gamma}\right|_{\partial K \backslash \gamma} \equiv 0$, where $m_{\gamma}$ is the midpoint of $\gamma \in \mathcal{E}_{K}$, and take $v=\theta_{\gamma}$ in (13). By the Simpson and midpoint rules, the left-hand side of (13) becomes

$$
\begin{aligned}
\int_{\partial K}\left(g_{K}-Q_{1} \frac{\partial u}{\partial n_{K}}\right) \theta_{\gamma} d s & =\frac{2}{3}|\gamma|\left(g_{K}-Q_{1} \frac{\partial u}{\partial n_{K}}\right)\left(m_{\gamma}\right) \\
& =\frac{2}{3} \int_{\gamma}\left(g_{K}-\frac{\partial u}{\partial n_{K}}\right) d s
\end{aligned}
$$

where $|\gamma|$ denotes the length of $\gamma$. To bound the right-hand side of (13), we employ the estimates

$$
\left\|\theta_{\gamma}\right\|_{K}+h_{K}^{-1 / 2}\left\|\theta_{\gamma}\right\|_{0, \gamma} \leq C, \quad\left\|w-Q_{1} w\right\|_{0, \gamma} \leq C h_{K}^{3 / 2}\|w\|_{2, K}
$$

to obtain

$$
\begin{gathered}
\left|B_{K}\left(\phi_{K}-e, \theta_{\gamma}\right)\right| \leq C\left\|e-\phi_{K}\right\|_{K}, \\
\left|\int_{\partial K}\left(\frac{\partial u}{\partial n_{K}}-Q_{1} \frac{\partial u}{\partial n_{K}}\right) \theta_{\gamma} d s\right| \leq C h_{K}^{2}\|u\|_{3, K} .
\end{gathered}
$$

Consequently, it follows that

$$
\left|\int_{\gamma}\left(g_{K}-\frac{\partial u}{\partial n_{K}}\right) d s\right| \leq C\left\|e-\phi_{K}\right\|_{K}+C h_{K}^{2}\|u\|_{3, K}
$$

The proof is done by applying the estimate (12) and summing over $\gamma \in \mathcal{E}_{K}$.

Under Condition $(\alpha, \sigma)$ on the triangulation $\mathcal{T}_{h}$, the following global superconvergence result was derived in $[22]$

$$
\left\|u_{h}-u_{I}\right\|_{\Omega} \leq C h^{1+\rho}\left(\|u\|_{3, \Omega}+|u|_{2, \infty, \Omega}\right)
$$


with $\rho=\min \left(\alpha, \frac{\sigma}{2}, \frac{1}{2}\right)$. Using this result, we are now able to derive the main result of this section.

Theorem 3.3. Assume that the triangulation $\mathcal{T}_{h}$ satisfies Condition $(\alpha, \sigma)$ and $u \in H^{3}(\Omega) \cap W^{2, \infty}(\Omega)$. Then there exists a constant $C>0$ such that

$$
\left\|\boldsymbol{\sigma}_{h}-\Pi_{h} \boldsymbol{\sigma}\right\|_{0, \Omega} \leq C h^{1+\rho}\left(\|u\|_{3, \Omega}+|u|_{2, \infty, \Omega}\right)
$$

with $\rho=\min \left(\alpha, \frac{\sigma}{2}, \frac{1}{2}\right)$.

Proof. At the beginning of this section, we observed that

$$
\left\|\boldsymbol{\sigma}_{h}-\Pi_{h} \boldsymbol{\sigma}\right\|_{0, \Omega}^{2} \leq C \sum_{K \in \mathcal{T}_{h}} \sum_{\gamma \in \mathcal{E}_{K}}\left|\int_{\gamma}\left(g_{K}-\frac{\partial u}{\partial n_{K}}\right) d s\right|^{2} .
$$

The sum over $K \in \mathcal{T}_{h}$ is split into two sums over $K \in \mathcal{T}_{0, h}$ and over the remaining elements. Lemma 3.2 yields the following bound for the first sum

$$
\sum_{K \in \mathcal{T}_{0, h}} \sum_{\gamma \in \mathcal{E}_{K}}\left|\int_{\gamma}\left(g_{K}-\frac{\partial u}{\partial n_{K}}\right) d s\right|^{2} \leq C\left\|u_{h}-u_{I}\right\|_{\Omega}^{2}+C h^{2(1+\min (\alpha, 1))}\|u\|_{3, \Omega}^{2},
$$

while Lemma 3.1 yields

$$
\sum_{K \in \mathcal{T}_{h} \backslash \mathcal{T}_{0, h}} \sum_{\gamma \in \mathcal{E}_{K}}\left|\int_{\gamma}\left(g_{K}-\frac{\partial u}{\partial n_{K}}\right) d s\right|^{2} \leq C\left\|u_{h}-u_{I}\right\|_{\Omega}^{2}+\sum_{K \in \mathcal{T}_{h} \backslash \mathcal{T}_{0, h}} C h_{K}^{2}|u|_{2, \omega_{K}}^{2} .
$$

Furthermore, we can obtain by (10)

$$
\sum_{K \in \mathcal{T}_{h} \backslash \mathcal{T}_{0, h}} C h_{K}^{2}|u|_{2, \omega_{K}}^{2} \leq C h^{2}\left(\sum_{K \in \mathcal{T}_{h} \backslash \mathcal{T}_{0, h}}\left|\omega_{K}\right|\right)|u|_{2, \infty, \Omega}^{2} \leq C h^{2+\min (\sigma, 1)}|u|_{2, \infty, \Omega}^{2} .
$$

The proof is completed by collecting the results above and invoking (14).

Remark 3.4. Theorem 3.3 requires the regularity of $u$ up to the boundary $\partial \Omega$. For subdomains $\Omega_{0} \subset \subset \Omega_{1} \subset \subset \Omega$ with $\Omega_{1} \subset \Omega_{1, h}$, we can derive an interior estimate analogous to Theorem 4.6 of [17] for $h$ small enough

$$
\left\|\boldsymbol{\sigma}_{h}-\Pi_{h} \boldsymbol{\sigma}\right\|_{0, \Omega_{0}} \leq C\left(\Omega_{0}, \Omega_{1}\right)\left\{h^{1+\rho}\left(\|u\|_{3, \Omega_{1}}+|u|_{2, \infty, \Omega_{1}}\right)+\left\|u-u_{h}\right\|_{0, \Omega_{1}}\right\}
$$

under the interior regularity $u \in H^{3}\left(\Omega_{1}\right) \cap W^{2, \infty}\left(\Omega_{1}\right)$.

\section{Postprocessing and asymptotic exactness}

To improve the guaranteed error bound (8), we consider the following postprocessing scheme

$$
\min _{\varphi_{h} \in P_{2}^{0}}\left\|\boldsymbol{\sigma}_{h}+\operatorname{curl} \varphi_{h}-\nabla u_{h}\right\|_{0, \Omega}
$$

with the space of trial functions $\varphi_{h}$ chosen as

$$
P_{2}^{0}:=\left\{\varphi_{h} \in H^{1}(\Omega):\left.\varphi_{h}\right|_{K} \in P_{2}^{0}(K) \text { for } K \in \mathcal{T}_{h} \text { and }\left.\varphi_{h}\right|_{\gamma}=0 \text { for } \gamma \in \mathcal{E}_{N}\right\} .
$$


It is easy to verify that (15) is reduced to the following variational problem: find $\psi_{h} \in P_{2}^{0}$ such that

$$
\left(\operatorname{curl} \psi_{h}, \operatorname{curl} \varphi_{h}\right)_{\Omega}=-\left(\boldsymbol{\sigma}_{h}-\nabla u_{h}, \operatorname{curl} \varphi_{h}\right)_{\Omega} \quad \forall \varphi_{h} \in P_{2}^{0} .
$$

The same scheme was also considered in $[8,10,20]$ but with global and local trial function spaces different from our choice $P_{2}^{0}$.

The advantage of employing the global quadratic space $P_{2}^{0}$ in (15) is two-fold. Firstly, the global matrix system arising from (16) is not only symmetric and positive definite but also well-conditioned (at least on quasi-uniform meshes) because it is locally equivalent to the scaled mass matrix by the inequalities

$$
c_{1}\left\|\varphi_{h}\right\|_{0, K} \leq h_{K}\left\|\operatorname{curl} \varphi_{h}\right\|_{0, K} \leq c_{2}\left\|\varphi_{h}\right\|_{0, K} \quad \forall \varphi_{h} \in P_{2}^{0}(K) .
$$

Thus the problem (16) can be efficiently solved by the conjugate gradient method even without any preconditioning. Numerical results indeed confirm that significant improvement on the error bound can be obtained with only a few cg iterations. Secondly, it will be shown in the following theorem that $\boldsymbol{\sigma}_{h}+\operatorname{curl} \psi_{h} \in \mathbb{B D M}_{1}$ converges to $\boldsymbol{\sigma}=\nabla u$ at a higher rate than $\nabla u_{h}$ under the conditions of Theorem 3.3.

Recall that the following assumptions were made on the boundary data: $u_{D}$ is continuous and piecewise linear over $\mathcal{E}_{D}$ and $g_{N}$ is piecewise constant over $\mathcal{E}_{N}$, so that

$$
\left.u_{h}\right|_{\Gamma_{D}}=u_{D},\left.\quad \Pi_{h}^{1} \boldsymbol{\sigma} \cdot \boldsymbol{n}\right|_{\Gamma_{N}}=\left.\Pi_{h} \boldsymbol{\sigma} \cdot \boldsymbol{n}\right|_{\Gamma_{N}}=\left.\boldsymbol{\sigma}_{h} \cdot \boldsymbol{n}\right|_{\Gamma_{N}}=g_{N} .
$$

More general data entail only higher order data oscillations of $u_{D}$ and $g_{N}$ and do not affect subsequent results if $u_{D}$ and $g_{N}$ are piecewise smooth.

Lemma 4.1. Let $\psi_{h} \in P_{2}^{0}$ be the solution of (16). Then $\psi_{h}$ is also the solution of the following minimization problem

$$
\min _{\varphi_{h} \in P_{2}^{0}}\left\|\boldsymbol{\sigma}_{h}+\operatorname{curl} \varphi_{h}-\boldsymbol{\sigma}\right\|_{0, \Omega}
$$

Proof. Using integration by parts, we obtain for all $\varphi \in P_{2}^{0}$

$$
\left(\nabla\left(u-u_{h}\right), \operatorname{curl} \varphi_{h}\right)_{\Omega}=\int_{\partial \Omega}\left(u-u_{h}\right) \operatorname{curl} \varphi_{h} \cdot \boldsymbol{n} d s=0 .
$$

The equation (16) then becomes

$$
\left(\operatorname{curl} \psi_{h}, \operatorname{curl} \varphi_{h}\right)_{\Omega}=-\left(\boldsymbol{\sigma}_{h}-\nabla u, \operatorname{curl} \varphi_{h}\right)_{\Omega} \quad \forall \varphi_{h} \in P_{2}^{0}
$$

which is equivalent to the minimization problem (17).

Theorem 4.2. Under the assumptions of Theorem 3.3, there exists a constant $C>0$ such that

$$
\left\|\boldsymbol{\sigma}_{h}+\operatorname{curl} \psi_{h}-\boldsymbol{\sigma}\right\|_{0, \Omega} \leq C h^{1+\rho}\left(\|u\|_{3, \Omega}+|u|_{2, \infty, \Omega}\right)
$$

with $\rho=\min \left(\alpha, \frac{\sigma}{2}, \frac{1}{2}\right)$. 
Proof. Because $\operatorname{div}\left(\Pi_{h}^{1} \boldsymbol{\sigma}-\Pi_{h} \boldsymbol{\sigma}\right)=0$, we have

$$
\left(\Pi_{h}^{1}-\Pi_{h}\right) \boldsymbol{\sigma}=\operatorname{curl} \varphi_{h}
$$

for some continuous piecewise quadratic function $\varphi_{h}$. Moreover, we may assume that $\varphi_{h} \in P_{2}^{0}$, since

$$
\int_{\gamma} \frac{\partial \varphi_{h}}{\partial t_{\gamma}} d s=\int_{\gamma}\left(\Pi_{h}^{1} \boldsymbol{\sigma}-\Pi_{h} \boldsymbol{\sigma}\right) \cdot \boldsymbol{n}_{\gamma} d s=0 \quad \forall \gamma \in \mathcal{E}_{h}
$$

and

$$
\frac{\partial \varphi_{h}}{\partial t_{\gamma}}=\left(\Pi_{h}^{1} \boldsymbol{\sigma}-\Pi_{h} \boldsymbol{\sigma}\right) \cdot \boldsymbol{n}_{\gamma}=0 \quad \forall \gamma \in \mathcal{E}_{N},
$$

where $\frac{\partial w}{\partial t_{\gamma}}$ denotes the tangential derivative of $w$ along $\gamma$. Therefore it follows by Lemma 4.1 and Theorem 3.3 that

$$
\begin{aligned}
\left\|\boldsymbol{\sigma}_{h}+\operatorname{curl} \psi_{h}-\boldsymbol{\sigma}\right\|_{0, \Omega} & \leq\left\|\boldsymbol{\sigma}_{h}+\operatorname{curl} \varphi_{h}-\boldsymbol{\sigma}\right\|_{0, \Omega} \\
& \leq\left\|\boldsymbol{\sigma}_{h}-\Pi_{h} \boldsymbol{\sigma}\right\|_{0, \Omega}+\left\|\Pi_{h}^{1} \boldsymbol{\sigma}-\boldsymbol{\sigma}\right\|_{0, \Omega} \\
& \leq C h^{1+\rho}\left(\|u\|_{3, \Omega}+|u|_{2, \infty, \Omega}\right)+C h^{2}\|u\|_{3, \Omega},
\end{aligned}
$$

which proves the desired result.

Obviously, $\boldsymbol{\sigma}_{h}+\operatorname{curl} \psi_{h}$ has continuous normal components across edges of $\mathcal{T}_{h}$ and satisfies the local conservation law (7), so that the following guaranteed error bound holds

$$
\begin{aligned}
\left\|u-u_{h}\right\|_{\Omega} \leq \eta:=\left\{\sum _ { K \in \mathcal { T } _ { h } } \left(\| \boldsymbol{\sigma}_{h}\right.\right. & +\operatorname{curl} \psi_{h}-\nabla u_{h} \|_{0, K} \\
& \left.\left.+\frac{h_{K}}{\pi}\left\|\operatorname{div} \boldsymbol{\sigma}_{h}+f-c u_{h}\right\|_{0, K}\right)^{2}\right\}^{1 / 2} .
\end{aligned}
$$

Using Theorem 4.2, we are now able to establish that the error estimator $\eta$ is asymptotically exact when the triangulation $\mathcal{T}_{h}$ satisfies Condition $(\alpha, \sigma)$ and the solution $u$ is regular enough.

Theorem 4.3. Under the assumptions of Theorem 3.3, we have

$$
\left\|\nabla\left(u-u_{h}\right)\right\|_{0, \Omega}=\eta+O\left(h^{1+\rho}\right)
$$

with $\rho=\min \left(\alpha, \frac{\sigma}{2}, \frac{1}{2}\right)$. Moreover, it holds that

$$
\left|\frac{\eta}{\left\|u-u_{h}\right\|_{\Omega}}-1\right|=O\left(h^{\min (\rho, \epsilon)}\right),
$$

provided that

$$
\left\|\nabla\left(u-u_{h}\right)\right\|_{0, \Omega} \geq C h, \quad\left\|c^{1 / 2}\left(u-u_{h}\right)\right\|_{0, \Omega} \leq C h^{1+\epsilon}
$$

for some $C>0$ and $\epsilon>0$. 
Proof. For the first contribution of $\eta$, Theorem 4.2 gives

$$
\begin{aligned}
& \left|\left\|\nabla\left(u-u_{h}\right)\right\|_{0, \Omega}-\left\|\boldsymbol{\sigma}_{h}+\operatorname{curl} \psi_{h}-\nabla u_{h}\right\|_{0, \Omega}\right| \\
\leq & \left\|\nabla\left(u-u_{h}\right)-\left(\boldsymbol{\sigma}_{h}+\operatorname{curl} \psi_{h}-\nabla u_{h}\right)\right\|_{0, \Omega} \leq C h^{1+\rho} .
\end{aligned}
$$

Moreover, the second contribution is of order $O\left(h^{2}\right)$ if $f$ and $c$ are locally smooth, because the local conservation law (7) gives

$$
\frac{h_{K}}{\pi}\left\|\operatorname{div} \boldsymbol{\sigma}_{h}+f-c u_{h}\right\|_{0, K}=\frac{h_{K}}{\pi}\left\|f-c u_{h}-{\overline{\left(f-c u_{h}\right)}}_{K}\right\|_{0, K}=O\left(h_{K}^{2}\right),
$$

where the Poincaré inequality $\left\|v-\bar{v}_{K}\right\|_{0, K} \leq C h_{K}\|\nabla v\|_{0, K}$ is used with $\bar{v}_{K}=$ $\frac{1}{|K|}(v, 1)_{K}$. This proves the first result.

By the first result and the assumption on $\left\|c^{1 / 2}\left(u-u_{h}\right)\right\|_{0, \Omega}$, it follows that

and thus

$$
\left\|u-u_{h}\right\|_{\Omega}=\eta+O\left(h^{1+\min (\rho, \epsilon)}\right)
$$

$$
\left|\frac{\eta}{\left\|u-u_{h}\right\|_{\Omega}}-1\right| \leq \frac{C h^{1+\min (\rho, \epsilon)}}{h}=C h^{\min (\rho, \epsilon)},
$$

which is exactly the second result.

Remark 4.4. The postprocessing scheme (15) was fully discussed in [8], but unlike ours, the trial functions were continuous piecewise linear polynomials on red-refined triangulations. Numerical results there showed that $\boldsymbol{\sigma}_{h}$ given in [7] and two red-refinements of triangulations along with $3 \mathrm{cg}$ iterations for solving (16) is particularly effective. However, asymptotic exactness is reached only through successive red refinements.

Remark 4.5. The choice $P_{2}^{0}$ in the postprocessing scheme (15) is based on the hierarchical splitting $\mathbb{B D M}_{1}=\mathbb{R T}_{0}+\operatorname{curl} P_{2}^{0}$. In view of the minimization problem (17), the error estimator $\eta$ has a very similar concept to the hierarchical error estimator studied in $[6,9,18]$.

On the other hand, the minimization problem (17) over the full space of continuous piecewise quadratic functions $\varphi_{h}$ is the same as the $\mathbb{B D M}_{1}$ mixed finite element method

$$
\min _{\boldsymbol{\tau}_{h} \in \mathbb{B D M}}\left\|\boldsymbol{\tau}_{h}-\boldsymbol{\sigma}\right\|_{0, \Omega} \quad \text { subject to the constraint } \operatorname{div} \boldsymbol{\tau}_{h}=\operatorname{div} \boldsymbol{\sigma}_{h} .
$$

This clearly provides a better vector approximation than $\boldsymbol{\sigma}_{h}+\operatorname{curl} \psi_{h}$ but at a higher computational cost.

\section{Numerical results}

In this section we report some numerical results to confirm the theoretical results of the preceding section and demonstrate the performance of the postprocessing scheme (15). Two examples are considered below for the Poisson equation with the homogeneous Dirichlet condition

$$
-\Delta u=f \text { in } \Omega \quad \text { and } \quad u=0 \text { on } \partial \Omega .
$$



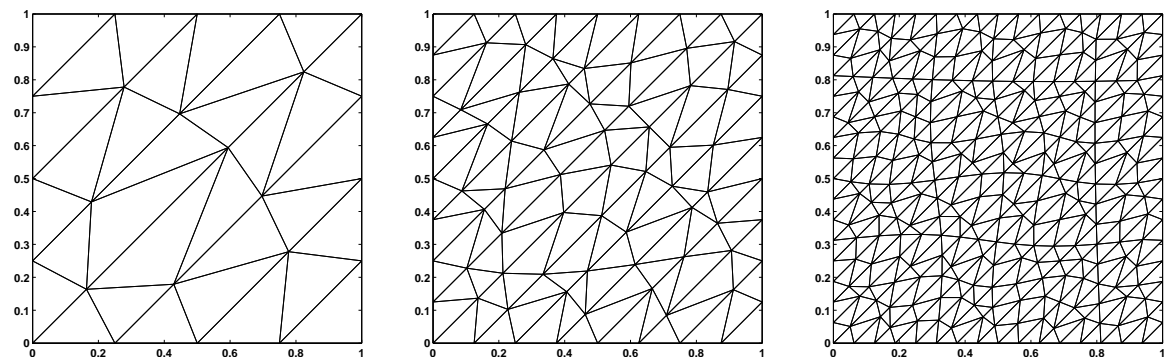

FiguRE 1. $O\left(h^{1.2}\right)$-perturbations of the uniform triangular meshes with the horizontal sizes $h=\frac{1}{4}, \frac{1}{8}, \frac{1}{16}$ for Example 1 .

The solution $u$ is smooth in the first example and has a corner singularity in the second example.

The matrix system (16) is approximately solved by applying the unpreconditioned conjugate gradient method with the zero starting vector. We will compare five error estimators computed with varying numbers of iterations. The first one is obtained by setting $\psi_{h}=0$ (i.e., no postprocessing), three are obtained by performing fixed numbers of iterations, $1,3,5$, and the last one is obtained by continuing the iteration until the relative residual norm is reduced to $10^{-10}$. These five error estimators will be denoted by $\eta_{i}$ for $i=0,1,3,5, \infty$.

Example 1. The domain is the unit square $\Omega=(0,1)^{2}$ on which the true solution is given by

$$
u(x, y)=\sin (2 \pi x)(1-\cos (2 \pi y))
$$

with the right-hand side $f=-\Delta u$. As the solution $u$ is smooth, we compute the finite element solutions on a sequence of quasi-uniform meshes generated by perturbing every interior vertex $(x, y)$ of the uniform triangular meshes with the horizontal sizes $h=\frac{1}{2^{m}}(2 \leq m \leq 10)$ by $(\delta x, \delta y)$, where

$$
\delta x=\delta y=0.5 h^{1.2} \sin \left(100 \pi^{2} x y\right) .
$$

These meshes clearly satisfy Condition $(\alpha, \sigma)$. Three meshes corresponding to the uniform ones with $h=\frac{1}{4}, \frac{1}{8}, \frac{1}{16}$ are shown in Fig. 1 .

Numerical results are reported in Fig. 2 which plots the effectivity indices

$$
\theta_{i}:=\frac{\eta_{i}}{\left\|\nabla\left(u-u_{h}\right)\right\|_{0, \Omega}} \quad(i=0,1,3,5, \infty) .
$$

Recall that the subscript $i$ refers to the number of $\mathrm{cg}$ iterations performed when solving the matrix system (16), with $i=\infty$ meaning that the iteration continues until the relative residual norm is reduced to $10^{-10}$. The actual error $\left\|\nabla\left(u-u_{h}\right)\right\|_{0, \Omega}$ is calculated by

$$
\left\|\nabla\left(u-u_{h}\right)\right\|_{0, \Omega}^{2}=\|\nabla u\|_{0, \Omega}^{2}-\left\|\nabla u_{h}\right\|_{0, \Omega}^{2},
$$




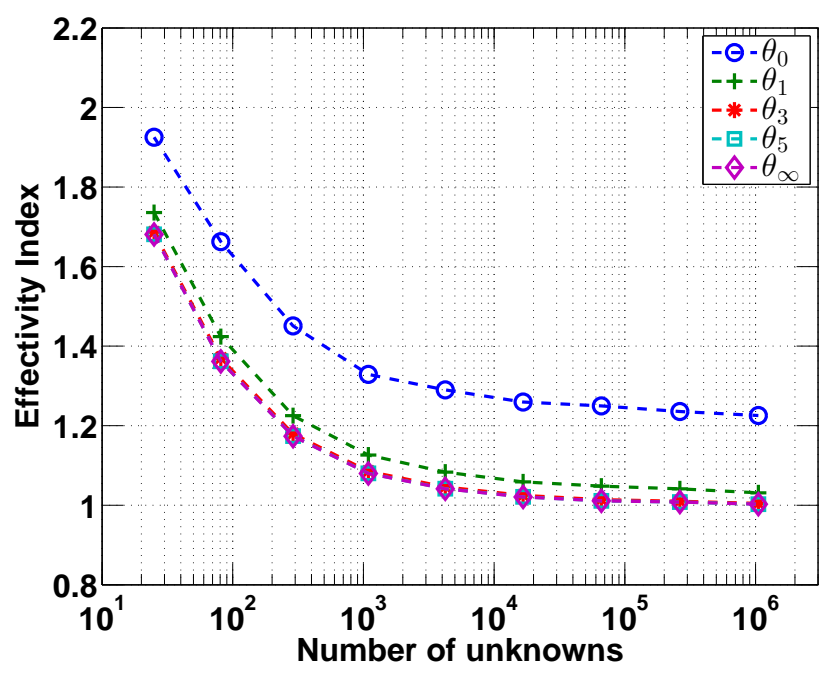

FiguRE 2. Effectivity indices $\theta_{i}=\frac{\eta_{i}}{\left\|\nabla\left(u-u_{h}\right)\right\|_{0, \Omega}}$ for Example 1.

where $\|\nabla u\|_{0, \Omega}^{2}=4 \pi^{2}$ is calculated analytically. For closer comparison we also report the numerical values of $\theta_{i}$ on the final mesh with the mesh size $h \approx \frac{1}{2^{10}}$ :

$$
\begin{gathered}
\theta_{0}=1.225668, \quad \theta_{1}=1.031096, \quad \theta_{3}=1.004631, \\
\theta_{5}=1.002676, \quad \theta_{\infty}=1.002672 .
\end{gathered}
$$

It can be seen that even a few number of cg iterations significantly improves the error estimator (more specifically, $\eta_{5}$ and $\eta_{\infty}$ are indistinguishable) and that $\eta_{\infty}$ (considered as solving exactly for $\psi_{h}$ ) seems asymptotically exact as predicted by Theorem 4.3.

Example 2. In this example the problem is posed on the L-shaped domain $\Omega=(-1,1)^{2} \backslash([0,1] \times[-1,0])$ with the right-hand side $f=1$. The true solution $u$ is unknown but the actual error $\left\|\nabla\left(u-u_{h}\right)\right\|_{0, \Omega}$ is calculated by

$$
\left\|\nabla\left(u-u_{h}\right)\right\|_{0, \Omega}^{2}=\|\nabla u\|_{0, \Omega}^{2}-\left\|\nabla u_{h}\right\|_{0, \Omega}^{2},
$$

where $\|\nabla u\|_{0, \Omega}^{2}=0.214075802680976$ is evaluated numerically (cf. [8]).

As the solution $u$ has a singularity at the origin, we perform adaptive mesh refinement starting with the initial mesh of six congruent triangles shown in the left of Fig. 3. The middle and right figures display two intermediate meshes generated by adaptive refinement. It is observed that the mesh refinement is concentrated around the singularity of the solution.

In Fig. 4 we plot the effectivity indices $\theta_{i}$ for $i=0,1,3,5, \infty$. The numerical values of $\theta_{i}$ 's on the final adapted mesh are given by

$$
\theta_{0}=1.304083, \quad \theta_{1}=1.039047, \quad \theta_{3}=1.003999,
$$



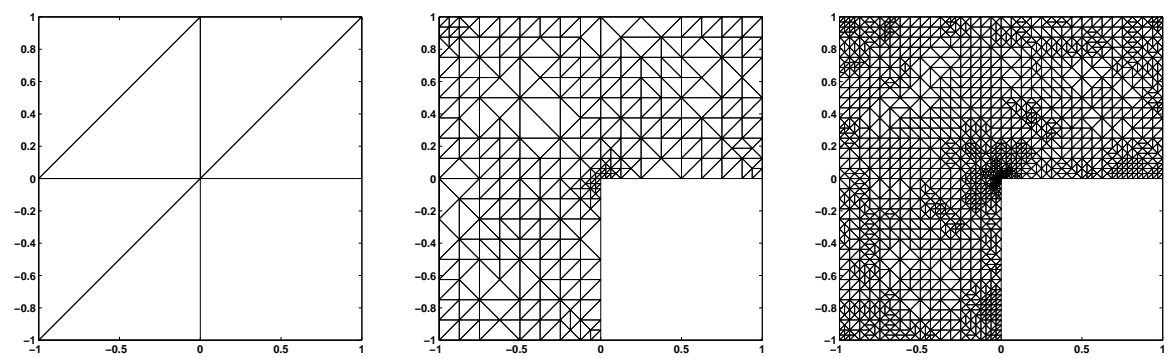

FIGURE 3. Initial mesh (left) and two intermediate meshes (middle and right) generated by the adaptive algorithm for Example 2.

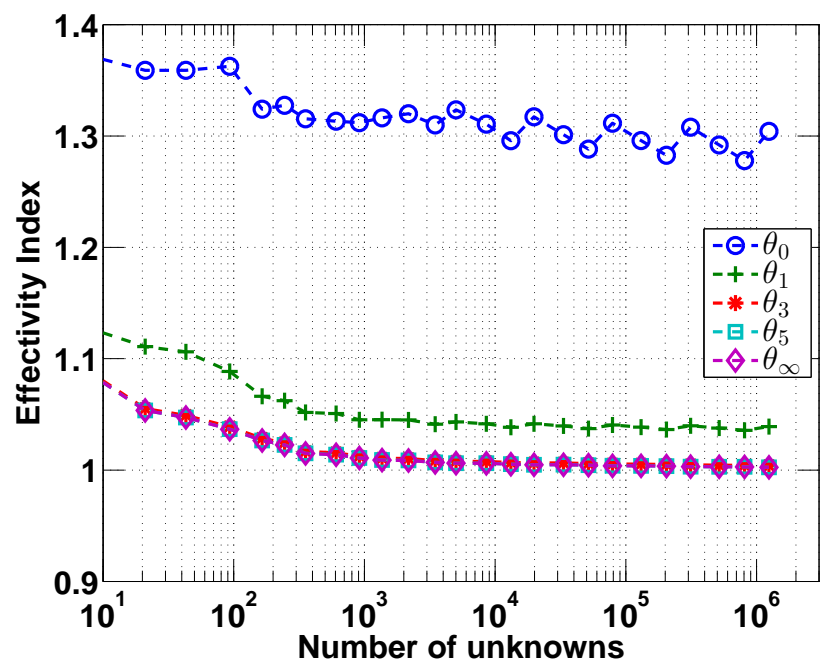

FIGURE 4. Effectivity indices $\theta_{i}=\frac{\eta_{i}}{\left\|\nabla\left(u-u_{h}\right)\right\|_{0, \Omega}}$ for Example 2.

$$
\theta_{5}=1.002506, \quad \theta_{\infty}=1.002474 .
$$

Note that the assumptions of Theorem 4.3 are violated; the solution $u$ is not regular enough and it is unclear whether the adapted meshes satisfy Condition $(\alpha, \sigma)$. Nevertheless, we observe the same phenomena as in the first example: (1) a few number of $\mathrm{cg}$ iterations are sufficient to significantly improve the error estimator, and (2) $\theta_{\infty}$ seems to approach one.

\section{References}

[1] M. Ainsworth, A framework for obtaining guaranteed error bounds for finite element approximations, J. Comput. Appl. Math. 234 (2010), no. 9, 2618-2632. 
[2] M. Ainsworth and J. T. Oden, A Posteriori Error Estimation in Finite Element Analysis, John Wiley \& Sons, New York, 2000.

[3] I. Babuška and T. Strouboulis, The Finite Element Method and Its Reliability, Oxford University Press, New York, 2001.

[4] R. E. Bank and J. Xu, Asymptotically exact a posteriori error estimators. I. grids with superconvergence, SIAM J. Numer. Anal. 41 (2003), no. 6, 2294-2312.

[5] - Asymptotically exact a posteriori error estimators. II. general unstructured grids, SIAM J. Numer. Anal. 41 (2003), no. 6, 2313-2332.

[6] F. A. Bornemann, B. Erdmann, and R. Kornhuber, A posteriori error estimates for elliptic problems in two and three space dimensions, SIAM J. Numer. Anal. 33 (1996), no. 3, 1188-1204

[7] D. Braess and J. Schöberl, Equilibrated residual error estimator for edge elements, Math. Comp. 77 (2008), no. 262, 651-672.

[8] C. Carstensen and C. Merdon, Effective postprocessing for equilibration a posteriori error estimators, Numer. Math. 123 (2013), no. 3, 425-459.

[9] P. Deuflhard, P. Leinen, and H. Yserentant, Concepts of an adaptive hierarchical finite element code, Impact Comput. Sci. Engrg. 1 (1989), no. 1, 3-35.

[10] P. Destuynder and B. Métivet, Explicit error bounds in a conforming finite element method, Math. Comp. 68 (1999), no. 228, 1379-1396.

[11] L. Du and N. Yan, Gradient recovery type a posteriori error estimate for finite element approximation on non-uniform meshes, Adv. Comput. Math. 14 (2001), no. 2, 175-193.

[12] R. Durán, M. A. Muschietti, and R. Rodríguez, On the asymptotic exactness of error estimators for linear triangular finite elements, Numer. Math. 59 (1991), no. 2, 107-127.

[13] R. Durán and R. Rodríguez, On the asymptotic exactness of Bank-Weiser's estimator, Numer. Math. 62 (1992), no. 3, 297-303.

[14] P. Ladevèze and D. Leguillon, Error estimate procedure in the finite element method and applications, SIAM J. Numer. Anal. 20 (1983), no. 3, 485-509.

[15] B. Li and Z. Zhang, Analysis of a class of superconvergence patch recovery techniques for linear and bilinear finite elements, Numer. Methods Partial Differential Equations 15 (1999), no. 2, 151-167.

[16] R. Luce and B. I. Wohlmuth, A local a posteriori error estimator based on equilibrated fluxes, SIAM J. Numer. Anal. 42 (2004), no. 4, 1394-1414.

[17] A. Maxim, Asymptotic exactness of an a posteriori error estimator based on the equilibrated residual method, Numer. Math. 106 (2007), no. 2, 225-253.

[18] J. S. Ovall, Function, gradient, and Hessian recovery using quadratic edge-bump functions, SIAM J. Numer. Anal. 45 (2007), no. 3, 1064-1080.

[19] S. Repin, S. Sauter, and A. Smolianski, A posteriori error estimation for the Poisson equation with mixed Dirichlet/Neumann boundary conditions, J. Comput. Appl. Math. 164/165 (2004), 601-612.

[20] T. Vejchodský, Guaranteed and locally computable a posteriori error estimate, IMA J. Numer. Anal. 26 (2006), no. 3, 525-540.

[21] M. Vohralík, Guaranteed and fully robust a posteriori error estimates for conforming discretizations of diffusion problems with discontinuous coefficients, J. Sci. Comput. 46 (2011), no. 3, 397-438

[22] J. Xu and Z. Zhang, Analysis of recovery type a posteriori error estimators for mildly structured grids, Math. Comp. 73 (2004), no. 247, 1139-1152.

[23] N. Yan and A. Zhou, Gradient recovery type a posteriori error estimates for finite element approximations on irregular meshes, Comput. Methods Appl. Mech. Engrg. 190 (2001), no. 32-33, 4289-4299. 
Department of Mathematics

KangWon National University

Chuncheon 200-701, Korea

E-mail address: eulerkim@kangwon.ac.kr 